\title{
Approximation properties of real and complex Post-Widder operators based on $q$-integers
}

Zafer Ünal, Mehmet Ali Özarslan, and Oktay Duman 


\title{
APPROXIMATION PROPERTIES OF REAL AND COMPLEX POST-WIDDER OPERATORS BASED ON $q$-INTEGERS
}

\author{
ZAFER ÜNAL, MEHMET ALI ÖZARSLAN, AND OKTAY DUMAN
}

Received 18 December, 2010

\begin{abstract}
In this paper, we study the statistical approximation properties of real and complex Post-Widder operators based on $q$-integers. We also obtain a Voronovskaya-type formula in statistical sense for these operators.
\end{abstract}

2000 Mathematics Subject Classification: 41A36, 40A35, 30E10

Keywords: $A$-statistical convergence, statistical approximation, Post-Widder operators, $q$-integers

\section{INTRODUCTION}

Positive linear operators and their $q$-modifications have recently become an active research area in the approximation theory (see, i.e., [1,2,6,7,10,12,13,16,18-26,28]). In this paper, we improve the classical Post-Widder operators via the techniques from the $q$-calculus. We obtain various statistical approximation theorems including some Korovkin-type results and Voronovskaya-type formulas in statistical and ordinary sense for these operators. Our investigations cover both real and complex cases.

Throughout the paper we employ the standard notations of $q$-calculus. As usual, by a $q$-integer and a $q$-factorial we mean respectively:

$$
[n]_{q}:=1+q+\ldots+q^{n} \text { for } n \in \mathbb{N} \text { with }[0]_{q}:=0
$$

and

$$
[n]_{q} !:=[1]_{q}[2]_{q} \ldots[n]_{q} \text { for } n \in \mathbb{N} \text { with }[0]_{q} !:=1 .
$$

Also, a $q$-binomial is defined by

$$
\left[\begin{array}{l}
n \\
k
\end{array}\right]_{q}:=\frac{[n]_{q} !}{[k]_{q} ![n-k]_{q} !} \text { for } 0 \leq k \leq n .
$$

Now let $0<q<1$. For simplicity, we use the following standard notations:

$$
(1+t)_{q}^{\infty}:=\prod_{j=0}^{\infty}\left(1+q^{j} t\right) .
$$


Then, we know $[14,15]$ that the $q$-Jackson definite integral of a function $f$ is defined by

$$
\int_{0}^{a} f(t) d_{q} t:=a(1-q) \sum_{n=0}^{\infty} f\left(a q^{n}\right) q^{n} \text { for } a>0 .
$$

Now we recall the $q$-analogue of the exponential function as follows (see $[3,17]$ ):

$$
E_{q}(x)=\sum_{n=0}^{\infty} q^{n(n-1) / 2} \frac{x^{n}}{[n]_{q} !}=(1+(1-q) x)_{q}^{\infty} .
$$

Notice that the above series converges for every $x$ whenever $q \in(0,1)$. Then, the corresponding $q$-Gamma function is defined by (see [17])

$$
\Gamma_{q}(x):=\int_{0}^{1 /(1-q)} t^{x-1} E_{q}(-q t) d_{q} t .
$$

As usual, let $C_{B}[0, \infty)$ denote the space of all bounded and continuous functions on $[0, \infty)$ endowed with the norm

$$
\|f\|=\sup _{x \in[0, \infty)}|f(x)| .
$$

In the present paper, we improve the classical Post-Widder operators by considering the above terminology as follows:

$$
P_{n, q}(f ; x)=\frac{1}{[n]_{q} !} \int_{0}^{1 /(1-q)} f\left(\frac{x t}{[n]_{q}}\right) t^{n} E_{q}(-q t) d_{q} t,
$$

where $x \in[0, \infty), q \in(0,1)$ and $f \in C_{B}[0, \infty)$. We use the following test functions

$$
e_{m}(y)=y^{m} \text { for } m \in \mathbb{N}_{0}:=\mathbb{N} \cup\{0\} .
$$

Then, we first observe that these operators are positive and linear and that

$$
P_{n, q}\left(e_{0} ; x\right)=e_{0}(x)=1 .
$$

Notice that the operators $P_{n, q}(f)$ map $C_{B}[0,+\infty)$ into itself. Also, it follows from (1.1) that

$$
\lim _{q \rightarrow 1^{-}} P_{n, q}(f ; x)=\frac{1}{n !} \int_{0}^{\infty} f\left(\frac{x t}{n}\right) t^{n} e^{-t} d t=: P_{n}(f ; x),
$$

which is the classical Post-Widder operator (see, for instance, [5, 27]). 


\section{LOCAl APPROXimation Results FOr $q$-POST-Widder Operators}

Let $f \in C_{B}[0, \infty)$ and $\delta>0$. Then, we consider the first modulus of continuity, the Peetre's $K$-functional, and the second modulus of smoothness, which are defined respectively by:

$$
\begin{gathered}
\omega(f, \delta)=\sup \{|f(y)-f(x)|:|y-x| \leq \delta ; x, y \in[0, \infty)\}, \\
K(f, \delta)=\inf \left\{\|f-g\|+\delta\left\|g^{\prime \prime}\right\|: g \in C_{B}^{2}[0, \infty)\right\}, \\
\omega_{2}(f, \delta)=\sup _{0<h \leq \delta} \sup _{x \in[0, \infty)}|f(x+2 h)-2 f(x+h)+f(x)|,
\end{gathered}
$$

where $C_{B}^{2}[0, \infty):=\left\{g \in C_{B}[0, \infty): g^{\prime}, g^{\prime \prime} \in C_{B}[0, \infty)\right\}$. Then, it is well-known that (see [4])

$$
K(f, \delta) \leq A \omega_{2}(f, \sqrt{\delta})
$$

for some positive constant $A$. With this terminology, in order to get a local approximation result for $q$-Post-Widder Operators we first need the following two results.

Lemma 1. For every $m, n \in \mathbb{N}$ and $x \in[0,+\infty)$, we have

$$
P_{n, q}\left(e_{m} ; x\right)=\frac{[n+1]_{q}[n+2]_{q} \ldots[n+m]_{q}}{[n]_{q}^{m}} x^{m} .
$$

Proof. Let $m, n \in N$ and $x \in[0,+\infty)$. Then, by (1.1) and (1.2), we get

$$
\begin{aligned}
P_{n, q}\left(e_{m} ; x\right) & =\frac{1}{[n]_{q} !} \int_{0}^{1 /(1-q)}\left(\frac{x t}{[n]_{q}}\right)^{m} t^{n} E_{q}(-q t) d_{q} t \\
& =\frac{1}{[n]_{q} !} \frac{x^{m}}{[n]_{q}^{m}} \int_{0}^{1 /(1-q)} t^{n+m} E_{q}(-q t) d_{q} t \\
& =\frac{1}{[n]_{q} !} \frac{x^{m}}{[n]_{q}^{m}} \Gamma_{q}(n+m+1) \\
& =\frac{1}{[n]_{q}^{m}} \frac{[n+m]_{q} !}{[n]_{q} !} x^{m} \\
& =\frac{[n+1]_{q}[n+2]_{q} \ldots[n+m]_{q}}{[n]_{q}^{m}} x^{m},
\end{aligned}
$$

which completes the proof.

If we use the moment function given by

$$
\varphi(y):=\varphi_{x}(y)=y-x,
$$

then the following result is an immediate consequence of Lemma 1. 
Corollary 1. For every $n \in \mathbb{N}$ and $x \in[0,+\infty)$, we have

$$
\begin{aligned}
P_{n, q}(\varphi ; x) & =\left(\frac{[n+1]_{q}}{[n]_{q}}-1\right) x, \\
P_{n, q}\left(\varphi^{2} ; x\right) & =\left(\frac{[n+1]_{q}[n+2]_{q}}{[n]_{q}^{2}}-\frac{2[n+1]_{q}}{[n]_{q}}+1\right) x^{2} .
\end{aligned}
$$

Now we are ready to give our approximation result.

Theorem 1. Let $q \in(0,1)$. Then, for every $n \in \mathbb{N}, x \in[0,+\infty)$ and $f \in C_{B}[0, \infty)$, we have

$$
\left|P_{n, q}(f ; x)-f(x)\right| \leq C \omega_{2}\left(f, x \delta_{n}\right)+\omega\left(f, x \alpha_{n}\right)
$$

for some positive constant $C$, where

$$
\delta_{n}:=\left(\frac{[n+1]_{q}^{2}}{[n]_{q}^{2}}+\frac{[n+1]_{q}[n+2]_{q}}{[n]_{q}^{2}}-\frac{4[n+1]_{q}}{[n]_{q}}+2\right)^{1 / 2}
$$

and

$$
\alpha_{n}:=\frac{[n+1]_{q}}{[n]_{q}}-1
$$

Proof. Define an auxiliary operator $P_{n, q}^{*}: C_{B}[0, \infty) \rightarrow C_{B}[0, \infty)$ by

$$
P_{n, q}^{*}(f ; x):=P_{n, q}(f ; x)-f\left(\frac{[n+1]_{q}}{[n]_{q}} x\right)+f(x) .
$$

Then, by Corollary 1, we get

$$
P_{n, q}^{*}(\varphi ; x)=0 .
$$

Now, for a given $g \in C_{B}^{2}[0, \infty)$, it follows from the Taylor formula that

$$
g(y)-g(x)=(y-x) g^{\prime}(x)+\int_{x}^{y}(y-u) g^{\prime \prime}(u) d u, \quad y \in[0, \infty) .
$$

Taking into account (2.3) and using (2.4) we get, for every $x \in[0, \infty)$, that

$$
\begin{aligned}
\left|P_{n, q}^{*}(g ; x)-g(x)\right| & =\left|P_{n, q}^{*}(g(y)-g(x) ; x)\right| \\
& =\left|g^{\prime}(x) P_{n, q}^{*}(\varphi ; x)+P_{n, q}^{*}\left(\int_{x}^{y}(y-u) g^{\prime \prime}(u) d u ; x\right)\right| \\
& =\left|P_{n, q}^{*}\left(\int_{x}^{y}(y-u) g^{\prime \prime}(u) d u ; x\right)\right|
\end{aligned}
$$




$$
\begin{aligned}
= & \mid P_{n, q}\left(\int_{x}^{y}(y-u) g^{\prime \prime}(u) d u ; x\right) \\
& -\int_{x}^{x[n+1]_{q} /[n]_{q}}\left(\frac{[n+1]_{q}}{[n]_{q}} x-u\right) g^{\prime \prime}(u) d u \mid .
\end{aligned}
$$

Since

$$
\left|P_{n, q}\left(\int_{x}^{y}(y-u) g^{\prime \prime}(u) d u ; x\right)\right| \leq \frac{\left\|g^{\prime \prime}\right\|}{2} P_{n, q}\left(\varphi^{2} ; x\right)
$$

and

$$
\left|\int_{x}^{x[n+1]_{q} /[n]_{q}}\left(\frac{[n+1]_{q}}{[n]_{q}} x-u\right) g^{\prime \prime}(u) d u\right| \leq \frac{\left\|g^{\prime \prime}\right\|}{2}\left(\frac{[n+1]_{q}}{[n]_{q}}-1\right)^{2} x^{2}
$$

we get

$$
\left|P_{n, q}^{*}(g ; x)-g(x)\right| \leq \frac{\left\|g^{\prime \prime}\right\|}{2} P_{n, q}\left(\varphi^{2} ; x\right)+\frac{\left\|g^{\prime \prime}\right\|}{2}\left(\frac{[n+1]_{q}}{[n]_{q}}-1\right)^{2} x^{2} .
$$

Hence, Corollary 1 implies that

$$
\begin{aligned}
& \quad\left|P_{n, q}^{*}(g ; x)-g(x)\right| \\
& \leq \frac{\left\|g^{\prime \prime}\right\|}{2}\left(\left(\frac{[n+1]_{q}[n+2]_{q}}{[n]_{q}^{2}}-\frac{2[n+1]_{q}}{[n]_{q}}+1\right)+\left(\frac{[n+1]_{q}}{[n]_{q}}-1\right)^{2}\right) x^{2},
\end{aligned}
$$

which gives

$$
\left|P_{n, q}^{*}(g ; x)-g(x)\right| \leq \frac{\left\|g^{\prime \prime}\right\|}{2}\left(\frac{[n+1]_{q}^{2}}{[n]_{q}^{2}}+\frac{[n+1]_{q}[n+2]_{q}}{[n]_{q}^{2}}-\frac{4[n+1]_{q}}{[n]_{q}}+2\right) x^{2} .
$$

Now, considering (2.1) and (2.2), if $f \in C_{B}[0, \infty)$ and $g \in C_{B}^{2}[0, \infty)$, we may write from (2.5) that

$$
\begin{aligned}
\left|P_{n, q}(f ; x)-f(x)\right| \leq & \left|P_{n, q}^{*}(f-g ; x)-(f-g)(x)\right| \\
& +\left|P_{n, q}^{*}(g ; x)-g(x)\right|+\left|f\left(\frac{[n+1]_{q}}{[n]_{q}} x\right)-f(x)\right| \\
\leq & 2\|f-g\|+x^{2} \delta_{n} \frac{\left\|g^{\prime \prime}\right\|}{2}+\left|f\left(\frac{[n+1]_{q}}{[n]_{q}} x\right)-f(x)\right| \\
\leq & 2\left(\|f-g\|+x^{2} \delta_{n}\left\|g^{\prime \prime}\right\|\right)+\omega\left(f, x \alpha_{n}\right),
\end{aligned}
$$


which yields that

$$
\begin{aligned}
\left|P_{n, q}(f ; x)-f(x)\right| & \leq 2 K\left(f, x^{2} \delta_{n}^{2}\right)+\omega\left(f, x \alpha_{n}\right) \\
& \leq C \omega_{2}\left(f, x \delta_{n}\right)+\omega\left(f, x \alpha_{n}\right) .
\end{aligned}
$$

Therefore, the proof is completed.

Now we consider the elements of locally Lipschitz functions. Let $0<\alpha \leq 1$ and let $D$ be a subset of the interval $[0, \infty)$. Then, by $\operatorname{Lip}_{M}(D, \alpha)$ we denote the space of all functions satisfying the condition

$$
|f(y)-f(x)| \leq M|y-x|^{\alpha} \text { for } y \in \bar{D} \text { and } x \in[0, \infty),
$$

where $\bar{D}$ denotes the closure of $D$ in $[0, \infty)$.

Then we can give our second local approximation result.

Theorem 2. Let $D$ be a subset of $[0, \infty), q \in(0,1)$ and $\alpha \in(0,1]$. Then, for every $n \in \mathbb{N}, x \in[0,+\infty)$ and $f \in C_{B}[0, \infty) \cap \operatorname{Lip}_{M}(D, \alpha)$, we have

$$
\left|P_{n, q}(f ; x)-f(x)\right| \leq M\left\{\gamma_{n}^{\alpha} x^{\alpha}+(d(x, D))^{\alpha}\right\},
$$

where $M$ is a positive constant; $d(x, D)$ is the distance between $x$ and $E$ defined as $d(x, D):=\inf \{|y-x|: y \in D\} ;$ and $\gamma_{n}$ is given by

$$
\gamma_{n}:=\left(\frac{[n+1]_{q}[n+2]_{q}}{[n]_{q}^{2}}-\frac{2[n+1]_{q}}{[n]_{q}}+1\right)^{1 / 2} .
$$

Proof. Let $\bar{D}$ denote the closure of $D$ in $[0, \infty)$. Let $x \in[0, \infty)$ be fixed. Then, there exists a point $x_{0} \in \bar{D}$ such that $\left|x-x_{0}\right|=d(x, D)$. Using the triangle inequality, we may write that

$$
|f(y)-f(x)| \leq\left|f(y)-f\left(x_{0}\right)\right|+\left|f(x)-f\left(x_{0}\right)\right| .
$$

Hence, it follows from (2.6) that

$$
\begin{aligned}
\left|P_{n, q}(f ; x)-f(x)\right| & \leq P_{n, q}(|f(y)-f(x)| ; x) \\
& \leq P_{n, q}\left(\left|f(y)-f\left(x_{0}\right)\right| ; x\right)+\left|f(x)-f\left(x_{0}\right)\right| \\
& \leq M\left\{P_{n, q}\left(\left|y-x_{0}\right|^{\alpha} ; x\right)+\left|x-x_{0}\right|^{\alpha}\right\} . \\
& =M\left\{P_{n, q}\left(\left|y-x_{0}\right|^{\alpha} ; x\right)+(d(x, D))^{\alpha}\right\} .
\end{aligned}
$$

Using the Cauchy-Bunyakowsky-Schwarz inequality for positive linear operators we find that

$$
\left|P_{n, q}(f ; x)-f(x)\right| \leq M\left\{P_{n, q}\left(\varphi^{2} ; x\right)^{\alpha / 2}+(d(x, D))^{\alpha}\right\} .
$$

Thus, the proof follows from (2.7) and Corollary 1. 


\section{RATES OF CONVERGENCE OF THE OPERATORS $P_{n, q}$}

Consider the following weighted space

$$
E:=\left\{f \in C[0, \infty):|f(x)| \leq M\left(1+x^{2}\right) \text { for some } M>0\right\} .
$$

Now, for a given $b>0$, by $\omega_{b}(f, \delta)$ we denote the usual modulus of continuity of $f$ on the closed interval $[0, b]$, which is defined to be

$$
\omega(f, \delta)_{[0, b]}:=\sup \{|f(y)-f(x)|:|y-x| \leq \delta ; x, y \in[0, b]\} .
$$

The next theorem gives the rate of convergence of the operators $P_{n, q}(f)$ to $f$ for all $f \in E$.

Theorem 3. For every $f \in E, b>0$ and $q \in(0,1)$, we have

$$
\left\|P_{n, q}(f)-f\right\|_{[0, b]} \leq C\left\{\gamma_{n}^{2}+\omega\left(f, \gamma_{n}\right)_{[0, b+1]}\right\},
$$

where $\gamma_{n}$ is given by (2.7); $C$ is a positive constant depending on $f, b$; and the symbol $\|\cdot\|_{[0, b]}$ denotes the classical sup-norm on the space $C[0, b]$.

Proof. Let $x \in[0, b]$ be fixed. Assume that $f \in E, b>0$ and $q \in(0,1)$. If $y \leq$ $b+1$, then one can write, for any $\delta>0$, that

$$
|f(y)-f(x)| \leq \omega(f,|y-x|)_{[0, b+1]} \leq\left(1+\frac{|y-x|^{2}}{\delta^{2}}\right) \omega(f, \delta)_{[0, b+1]} .
$$

On the other hand, if $y>b+1$, since $y-x>1$, we get, for some positive constant $M$ depending on $f \in E$, that

$$
\begin{aligned}
|f(y)-f(x)| & \leq M\left\{2+x^{2}+y^{2}\right\} \\
& =M\left\{2+x^{2}+((y-x)+x)^{2}\right\} \\
& =M\left\{2+2 x^{2}+2 x(y-x)+(y-x)^{2}\right\} \\
& \leq M\left\{2+2 x^{2}+(2 x+1)(y-x)^{2}\right\} \\
& \leq M\left(2 x^{2}+2 x+3\right)(y-x)^{2},
\end{aligned}
$$

which implies that

$$
|f(y)-f(x)| \leq N(y-x)^{2},
$$

where $N:=M\left(2 b^{2}+2 b+3\right)$. From (3.2) and (3.3), we get, for all $x \in[0, b]$ and $y \geq 0$, that

$$
|f(y)-f(x)| \leq N(y-x)^{2}+\left(1+\frac{(y-x)^{2}}{\delta^{2}}\right) \omega(f, \delta)_{[0, b+1]}
$$

and therefore

$$
\left|P_{n, q}(f ; x)-f(x)\right| \leq N P_{n, q}\left(\varphi^{2} ; x\right)+\omega(f, \delta)_{[0, b+1]}\left(1+\frac{1}{\delta^{2}} P_{n, q}\left(\varphi^{2} ; x\right)\right) .
$$


Now applying Corollary 1 , we obtain that

$$
\begin{aligned}
\left|P_{n, q}(f ; x)-f(x)\right| & \leq N \gamma_{n}^{2} x^{2}+\omega(f, \delta)_{[0, b+1]}\left(1+\frac{\gamma_{n}^{2} x^{2}}{\delta^{2}}\right) \\
& \leq N b^{2} \gamma_{n}^{2}+\omega_{b+1}(f, \delta)_{[0, b+1]}\left(1+\frac{\gamma_{n}^{2} b^{2}}{\delta^{2}}\right)
\end{aligned}
$$

where $\gamma_{n}$ is given by (2.7). If we take $\delta=\gamma_{n}$ on the right hand side of the last inequality, then we immediately see that

$$
\left|P_{n, q}(f ; x)-f(x)\right| \leq C\left\{\gamma_{n}^{2}+\omega\left(f, \gamma_{n}\right)_{[0, b+1]}\right\},
$$

where $C:=\max \left\{N b^{2}, 1+b^{2}\right\}$. Finally, taking supremum over $x \in[0, b]$, the proof is completed.

\section{STATISTICAL APPROXIMATION RESUlTS}

In this section, we study the statistical approximation properties of the $q$-PostWidder operators. We first recall the concept of statistical convergence.

Let $A:=\left[a_{j n}\right], j, n \in \mathbb{N}$, be an infinite matrix. The $A$-transform of a sequence $x:=\left(x_{n}\right)$ is given by $A x:=\left((A x)_{n}\right)$ with $(A x)_{n}=\sum_{n=1}^{\infty} a_{j n}$ provided that the series converges for each $n \in \mathbb{N}$. Then, we say that a matrix $A$ is regular if $\lim A x=L$ whenever $\lim x=L$. Now let $A=\left[a_{j n}\right]$ be nonnegative regular summability matrix. Then, a sequence $x=\left(x_{n}\right)$ is said to be $A$-statistically convergent to $L$ if $\lim _{n} \sum_{n:\left|x_{n}-L\right| \geq \varepsilon} a_{j n}=0$ holds for every $\varepsilon>0$. In this case, we write $s t_{A}-\lim x=$ $L$ (see [9]). It is well-known that if we take $A=C_{1}=\left[c_{j n}\right]$, the Cesáro matrix of order one defined to be $c_{j n}=1 / j$ if $1 \leq n \leq j$, and $c_{j n}=0$ otherwise, then we get the classical definition of statistical convergence (see [8]). In this case, we use the notation $s t-\lim$ instead of $s t_{C_{1}}-\lim$. Also, if $A=I$, the identity matrix, then it reduces to the ordinary ordinary convergence. Notice that every convergent sequence is $A$-statistically convergent to the same value, but the converse does not hold true. As we can see the following example, there exists an $A$-statistically convergent sequence but non-convergent in the usual sense.

Now let $A=\left[a_{j n}\right]$ be a nonnegative regular summability matrix. Assume that $\left(q_{n}\right)$ is sequence from $(0,1)$ such that

$$
s t_{A}-\lim _{n} \frac{1}{[n]_{q_{n}}}=0, s t_{A}-\lim _{n} q_{n}=1 \text { and } s t_{A}-\lim _{n} q_{n}^{n}=\beta \in(0,1) .
$$

Indeed, such a sequence $\left(q_{n}\right)$ can be constructed by the following way. Take $A=$ $C_{1}=\left[c_{j n}\right]$ and define the sequence $\left(q_{n}\right)$ by

$$
q_{n}:= \begin{cases}e^{-n}, & \text { if } n=m^{2}(m \in \mathbb{N}) \\ \frac{n}{n+1}, & \text { otherwise }\end{cases}
$$


Then observe that $q_{n} \in(0,1)$ for each $n \in \mathbb{N}$. It is easy to check that although $\left(q_{n}\right)$ is non-convergent in the usual sense, it satisfies the conditions in (4.1) with the choice of $A=C_{1}$ and $\beta=1 / e$. On the other hand, since

$$
\frac{[n+1]_{q_{n}}}{[n]_{q_{n}}}=\frac{1-q_{n}^{n}}{1-q_{n}^{n-1}} \text { and } \frac{[n+1]_{q_{n}}[n+2]_{q_{n}}}{[n]_{q_{n}}^{2}}=\frac{\left(1-q_{n}^{n}\right)\left(1-q_{n}^{n+1}\right)}{\left(1-q_{n}^{n-1}\right)^{2}},
$$

we obtain from (4.1) that

$$
s t_{A}-\lim _{n} \frac{[n+1]_{q_{n}}}{[n]_{q_{n}}}=s t_{A}-\lim _{n} \frac{[n+1]_{q_{n}}^{2}}{[n]_{q_{n}}^{2}}=s t_{A}-\lim _{n} \frac{[n+1]_{q_{n}}[n+2]_{q_{n}}}{[n]_{q_{n}}^{2}}=1 .
$$

Then it follows from (4.3) that

$$
s t_{A}-\lim _{n} \delta_{n}=s t_{A}-\lim \alpha_{n}=s t_{A}-\lim \gamma_{n}=0,
$$

where $\delta_{n}, \alpha_{n}$ and $\gamma_{n}$ are given by (2.1), (2.2) and (2.7), respectively.

Now taking a sequence $\left(q_{n}\right)$ from $(0,1)$ satisfying $(4.1)$ instead of a fixed number $q \in(0,1)$ in the definition of operators $(1.1)$, we obtain the following (pointwise) statistical approximation result.

Theorem 4. Let $A=\left[a_{j n}\right]$ be a nonnegative regular summability matrix, and let $\left(q_{n}\right)$ be a sequence from $(0,1)$ satisfying $(4.1)$. Then, for every $x \in[0,+\infty)$ and $f \in C_{B}[0, \infty)$, we have

$$
s t_{A}-\lim _{n}\left|P_{n, q_{n}}(f ; x)-f(x)\right|=0 .
$$

Proof. Let $x \in[0, \infty)$ and $f \in C_{B}[0, \infty)$ be fixed. By the right continuity of $\omega$ and $\omega_{2}$ at zero, one can get from (4.4) that

$$
s t_{A}-\lim _{n} \omega\left(f, x \alpha_{n}\right)=s t_{A}-\lim _{n} \omega_{2}\left(f, x \delta_{n}\right)=0 .
$$

Now, for a given $\varepsilon>0$, define the following sets:

$$
\begin{aligned}
U & :=\left\{n \in \mathbf{N}:\left|P_{n, q_{n}}(f ; x)-f(x)\right| \geq \varepsilon\right\}, \\
U_{1} & :=\left\{n \in \mathbf{N}: \omega\left(f ; x \alpha_{n}\right) \geq \frac{\varepsilon}{2}\right\}, \\
U_{2} & :=\left\{n \in \mathbf{N}: \omega_{2}\left(f ; x \delta_{n}\right) \geq \frac{\varepsilon}{2 C}\right\},
\end{aligned}
$$

where $C$ is a positive constant as in Theorem 1. Then, we may write from the inequality in Theorem 1 that

$$
U \subseteq U_{1} \cup U_{2}
$$

which gives, for every $j \in \mathbb{N}$,

$$
\sum_{n \in U} a_{j n} \leq \sum_{n \in U_{1}} a_{j n}+\sum_{n \in U_{2}} a_{j n}
$$


Letting $j \rightarrow \infty$ in (4.6) and also using (4.5), we have

$$
\lim _{j} \sum_{n \in U} a_{j n}=0
$$

which guarantees that

$$
s t_{A}-\lim _{n}\left|P_{n, q_{n}}(f ; x)-f(x)\right|=0 .
$$

Thus, the proof is completed.

If we take $A=I$, the identity matrix, by Theorem 4, we obtain the classical approximation result.

Corollary 2. Let $\left(q_{n}\right)$ be a sequence from $(0,1)$ satisfying the conditions:

$$
\lim _{n} \frac{1}{[n]_{q_{n}}}=0, \lim _{n} q_{n}=1 \text { and } \lim _{n} q_{n}^{n}=\eta \in(0,1) .
$$

Then, for every $x \in[0,+\infty)$ and $f \in C_{B}[0, \infty)$, the sequence $\left\{P_{n, q_{n}}(f ; x)\right\}$ converges (pointwise) to $f(x)$.

We should note that if a sequence $\left(q_{n}\right)$ satisfies (4.7), then it also satisfies (4.1); and hence the classical (pointwise) approximation result in Corollary 2 implies the statistical one in Theorem 4. However, if we choose the sequence $\left(q_{n}\right)$ as in (4.2), then we can easily see that (4.7) does not hold true while (4.1) is still valid. This example shows that our statistical approximation result in Theorem 4 is more applicable than the classical one.

Now we obtain a uniform approximation theorem in statistical sense.

Theorem 5. Let $A=\left[a_{j n}\right]$ be a nonnegative regular summability matrix, and let $\left(q_{n}\right)$ be a sequence from $(0,1)$ satisfying $(4.1)$. Assume that $D$ is a compact set in $[0, \infty)$. Then, for every $f \in C_{B}[0, \infty) \cap \operatorname{Lip} p_{M}(D, \alpha)$ with $M>0$ and $\alpha \in(0,1]$, we have

$$
s t_{A}-\lim _{n}\left\|P_{n, q_{n}}(f)-f\right\|_{D}=0 .
$$

Proof. Let $x \in D$ be fixed. In this case, we have $d(x, D)=0$. Using this and Theorem 2, we immediately get that, for each $x \in D$,

$$
\left|P_{n, q_{n}}(f ; x)-f(x)\right| \leq M \gamma_{n}^{\alpha} x^{\alpha} .
$$

Since $D$ is compact, the number $L:=\sup _{x \in D}\left\{x^{\alpha}\right\}$ is finite. Hence, taking supremum over $x \in D$ on the both sides of (4.8), we obtain that

$$
\left\|P_{n, q_{n}}(f)-f\right\|_{D} \leq L M \gamma_{n}^{\alpha}
$$

which yields, for every $\varepsilon>0$,

$$
\left\{n \in \mathbf{N}:\left\|P_{n, q_{n}}(f)-f\right\|_{D} \geq \varepsilon\right\} \subseteq\left\{n \in \mathbf{N}: \gamma_{n} \geq\left(\frac{\varepsilon}{L M}\right)^{1 / \alpha}\right\}
$$


and hence

$$
\sum_{n:\left\|P_{n, q_{n}}(f)-f\right\|_{D} \geq \varepsilon} a_{j n} \leq \sum_{n: \gamma_{n} \geq\left(\frac{\varepsilon}{L M}\right)^{1 / \alpha}} a_{j n}
$$

holds for every $j \in \mathbb{N}$. Now taking limit as $j \rightarrow \infty$ on both sides of the last inequality, and also considering (4.4), we deduce that

$$
\lim _{n:\left\|P_{n, q_{n}}(f)-f\right\|_{D} \geq \varepsilon} a_{j n}=0
$$

which is the desired result.

If $A=I$ in Theorem 5, then we easily get the uniform approximation result in the ordinary sense.

Corollary 3. Let $\left(q_{n}\right)$ be a sequence from $(0,1)$ satisfying $(4.7)$, and let $D$ be a compact set in $[0, \infty)$. Then, for every $f \in C_{B}[0, \infty) \cap \operatorname{Lip} p_{M}(D, \alpha)$ with $M>0$ and $\alpha \in(0,1]$, the sequence $\left\{P_{n, q_{n}}(f)\right\}$ is uniformly convergent to $f$ on $D$.

For a uniform approximation process, we also get the next result.

Theorem 6. Let $A=\left[a_{j n}\right]$ be a nonnegative regular summability matrix, and let $\left(q_{n}\right)$ be a sequence from $(0,1)$ satisfying $(4.1)$. Then, for every $f \in E$ and $b>0$, we have

$$
s t_{A}-\lim _{n}\left\|P_{n, q_{n}}(f)-f\right\|_{[0, b]}=0 .
$$

Proof. Assume that $x \in[0, b]$ is fixed. Then, for a given $\varepsilon>0$, consider the following sets:

$$
\begin{aligned}
V & :=\left\{n \in \mathbf{N}:\left\|P_{n, q_{n}}(f)-f\right\|_{[0, b]} \geq \varepsilon\right\}, \\
V_{1} & :=\left\{n \in \mathbf{N}: \gamma_{n} \geq\left(\frac{\varepsilon}{2 C}\right)^{1 / 2}\right\}, \\
V_{2} & :=\left\{n \in \mathbf{N}: \omega\left(f, \gamma_{n}\right)_{[0, b+1]} \geq \frac{\varepsilon}{2 C}\right\},
\end{aligned}
$$

where $\gamma_{n}$ is given by (2.7), and $C$ is a positive constant as in Theorem 3. Then, it follows from Theorem 3 that

$$
V \subseteq V_{1} \cup V_{2}
$$

The last inclusion implies that

$$
\sum_{n \in V} a_{j n} \leq \sum_{n \in V_{1}} a_{j n}+\sum_{n \in V_{2}} a_{j n} .
$$

On the other hand, by the right continuity of $\omega(f,)_{[0, b+1]}$ at zero, one can get from (4.4) that

$$
s t_{A}-\lim _{n} \omega\left(f, \gamma_{n}\right)_{[0, b+1]}=0 .
$$


Then, letting $j \rightarrow \infty$ in (4.9) and using (4.4), (4.10), we easily get that

$$
\lim _{n} \sum_{n \in V} a_{j n}=0
$$

which completes the proof.

Of course, if we take $A=I$ in Theorem 6, we obtain the next result.

Corollary 4. Let $\left(q_{n}\right)$ be a sequence from $(0,1)$ satisfying $(4.7)$. Then, for every $f \in E$ and $b>0$, the sequence $\left\{P_{n, q_{n}}(f)\right\}$ is uniformly convergent to $f$ on $[0, b]$.

\section{VORONOVSKAYA-TYPE RESULTS}

In this section, we prove a Voronovskaya-type theorem for the $q$-Post-Widder operators. Throughout this section we consider a sequence $\left(q_{n}\right)$ satisfying (4.1) instead of a fixed $q \in(0,1)$ in the definition (1.1).

We first need the following lemma.

Lemma 2. Let $A=\left[a_{j n}\right]$ be a nonnegative regular summability matrix, and let $\left(q_{n}\right)$ be a sequence from $(0,1)$ satisfying $(4.1)$. Then, we have

$$
\begin{array}{rlr}
s t_{A}-\lim _{n \rightarrow \infty}[n]_{q_{n}} P_{n, q_{n}}(\varphi ; x) & =\beta x, \\
s t_{A}-\lim _{n \rightarrow \infty}[n]_{q_{n}} P_{n, q_{n}}\left(\varphi^{2} ; x\right) & =\beta x^{2}, \\
s t_{A}-\lim _{n \rightarrow \infty}[n]_{q_{n}}^{2} P_{n, q_{n}}\left(\varphi^{4} ; x\right) & = & 3 \beta^{2}(1-\beta) x^{4}
\end{array}
$$

uniformly with respect to $x \in[0, b],(b>0)$, where $\beta:=s t_{A}-\lim q_{n}^{n}$ as stated in (4.1).

Proof. It follows from Corollary 1 that

$$
[n]_{q_{n}} P_{n, q_{n}}(\varphi ; x)=\left([n+1]_{q_{n}}-[n]_{q_{n}}\right) x
$$

and

$$
[n]_{q_{n}} P_{n, q_{n}}\left(\varphi^{2} ; x\right)=\left(\frac{[n+1]_{q_{n}}[n+2]_{q_{n}}}{[n]_{q_{n}}}-2[n+1]_{q_{n}}+[n]_{q_{n}}\right) x^{2} .
$$

Then, using (4.1) we easily get

$$
s t_{A}-\lim _{n \rightarrow \infty}[n]_{q_{n}} P_{n, q_{n}}(\varphi ; x)=\beta x
$$

and

$$
s t_{A}-\lim _{n \rightarrow \infty}[n]_{q_{n}} P_{n, q_{n}}\left(\varphi^{2} ; x\right)=\beta x^{2}
$$

uniformly with respect to $x \in[0, b]$. On the other hand, by Lemma 1 , we obtain that

$$
\begin{aligned}
P_{n, q_{n}}\left(\varphi^{4} ; x\right)= & P_{n, q_{n}}\left(e_{4} ; x\right)-4 x P_{n, q_{n}}\left(e_{3} ; x\right)+6 x^{2} P_{n, q_{n}}\left(e_{2} ; x\right) \\
& -4 x^{3} P_{n, q_{n}}\left(e_{1} ; x\right)+x^{4}
\end{aligned}
$$




$$
\begin{aligned}
= & \frac{[n+1]_{q_{n}}[n+2]_{q_{n}}[n+3]_{q_{n}}[n+4]_{q_{n}}}{[n]_{q_{n}}^{4}} x^{4} \\
& -4 \frac{[n+1]_{q_{n}}[n+2]_{q_{n}}[n+3]_{q_{n}}}{[n]_{q_{n}}^{3}} x^{4} \\
& +6 \frac{[n+1]_{q_{n}}[n+2]_{q_{n}}}{[n]_{q_{n}}^{2}} x^{4}-4 \frac{[n+1]_{q_{n}}}{[n]_{q_{n}}} x^{4}+x^{4} .
\end{aligned}
$$

Then, we get

$$
\begin{aligned}
{[n]_{q_{n}}^{2} P_{n, q_{n}}\left(\varphi^{4} ; x\right)=} & \left(\frac{[n+1]_{q_{n}}[n+2]_{q_{n}}[n+3]_{q_{n}}[n+4]_{q_{n}}}{[n]_{q_{n}}^{2}}\right. \\
& -4 \frac{[n+1]_{q_{n}}[n+2]_{q_{n}}[n+3]_{q_{n}}}{[n]_{q_{n}}} \\
& \left.+6[n+1]_{q_{n}}[n+2]_{q_{n}}-4[n]_{q_{n}}[n+1]_{q_{n}}+[n]_{q_{n}}^{2}\right) x^{4} .
\end{aligned}
$$

Now using the fact that

$$
[n]_{q_{n}}=\frac{1-q_{n}^{n-1}}{1-q_{n}}
$$

and also considering (4.1), after some simple calculations, for each $x \in[0, \infty)$, we have

$$
s t_{A}-\lim _{n \rightarrow \infty}[n]_{q_{n}}^{2} P_{n, q_{n}}\left(\varphi^{4} ; x\right)=3 \beta^{2}(1-\beta) x^{4},
$$

uniformly with respect to $x \in[0, b]$, which is the desired result.

Then we get our Voronovskaya-type theorem as follows.

Theorem 7. Let $A=\left[a_{j n}\right]$ be a nonnegative regular summability matrix, and let $\left(q_{n}\right)$ be a sequence from $(0,1)$ satisfying (4.1). Then, for every $f \in E$ such that $f^{\prime}$, $f^{\prime \prime} \in E$, we have

$$
s t_{A}-\lim _{n \rightarrow \infty}[n]_{q_{n}}\left\{P_{n, q_{n}}(f ; x)-f(x)\right\}=\beta x f^{\prime}(x)+\frac{\beta x^{2} f^{\prime \prime}(x)}{2}
$$

uniformly with respect to $x \in[0, b],(b>0)$, where $\beta:=s t_{A}-\lim q_{n}^{n}$ as stated in (4.1).

Proof. Let $f, f^{\prime}, f^{\prime \prime} \in E$. For each $x \geq 0$, define a function $\Psi(y):=\Psi(y, x)$ by

$$
\Psi(y)= \begin{cases}\frac{f(y)-f(x)-(y-x) f^{\prime}(x)-\frac{1}{2}(y-x)^{2} f^{\prime \prime}(x)}{(y-x)^{2}}, & \text { if } y \neq x \\ 0, & \text { if } y=x .\end{cases}
$$


Then by assumption we have $\Psi(x, x)=0$ and the function $\Psi(\cdot, x)$ belongs to $E$. Hence, by Taylor's theorem we get

$$
f(y)=f(x)+(y-x) f^{\prime}(x)+\frac{(y-x)^{2}}{2} f^{\prime \prime}(x)+(y-x)^{2} \Psi(y, x) .
$$

Hence we easily observe that

$$
\begin{aligned}
{[n]_{q_{n}}\left\{P_{n, q_{n}}(f ; x)-f(x)\right\}=} & f^{\prime}(x)[n]_{q_{n}} P_{n, q_{n}}(\varphi ; x) \\
& +\frac{f^{\prime \prime}(x)}{2}[n]_{q_{n}} P_{n, q_{n}}\left(\varphi^{2} ; x\right) \\
& +[n]_{q_{n}} P_{n, q_{n}}\left(\varphi^{2} \Psi ; x\right) .
\end{aligned}
$$

If we apply the Cauchy-Schwarz inequality for the last term on the right-hand side of the last equality, then we conclude that

$$
[n]_{q_{n}}\left|P_{n, q_{n}}\left(\varphi^{2} \Psi ; x\right)\right| \leq\left([n]_{q_{n}}^{2} P_{n, q_{n}}\left(\varphi^{4} ; x\right)\right)^{1 / 2}\left(P_{n, q_{n}}\left(\Psi^{2} ; x\right)\right)^{1 / 2} .
$$

Let $\eta(y, x):=\Psi^{2}(y, x)$. In this case, observe that $\eta(x, x)=0$ and $\eta(\cdot, x) \in E$. Then, it follows from Theorem 6 that

$$
s t_{A}-\lim _{n \rightarrow \infty} P_{n, q_{n}}\left(\Psi^{2} ; x\right)=s t_{A}-\lim _{n \rightarrow \infty} P_{n, q_{n}}(\eta(y, x) ; x)=\eta(x, x)=0
$$

uniformly with respect to $x \in[0, b],(b>0)$. Now, by (5.1), (5.2), and Lemma 2, we obtain that

$$
s t_{A}-\lim _{n \rightarrow \infty}[n]_{q_{n}} P_{n, q_{n}}\left(\varphi^{2} \Psi ; x\right)=0
$$

uniformly with respect to $x \in[0, b]$. Combining the above facts with Lemma 2, we observe that

$$
s t_{A}-\lim _{n \rightarrow \infty}[n]_{q_{n}}\left\{P_{n, q_{n}}(f ; x)-f(x)\right\}=\beta x f^{\prime}(x)+\frac{\beta x^{2} f^{\prime \prime}(x)}{2}
$$

uniformly with respect to $x \in[0, b]$. Thus, the proof is completed.

The next result is an immediate consequence of Theorem 7 .

Corollary 5. Let $\left(q_{n}\right)$ be a sequence from $(0,1)$ satisfying (4.7). Then, for every $f \in E$ such that $f^{\prime}, f^{\prime \prime} \in E$, we have

$$
\lim _{n \rightarrow \infty}[n]_{q_{n}}\left\{P_{n, q_{n}}(f ; x)-f(x)\right\}=\eta x f^{\prime}(x)+\frac{\eta x^{2} f^{\prime \prime}(x)}{2}
$$

uniformly with respect to $x \in[0, b],(b>0)$, where $\eta=\lim q_{n}^{n}$ as stated in (4.1). 


\section{APPROXIMATION PROPERTIES OF COMPLEX $q$-POST-WIDDER OPERATORS}

In this section, we will introduce the complex $q$-Post-Widder operators and study their approximation properties.

Now, let

$$
D:=\{z \in \mathbb{C}:|z|<1\}, \bar{D}:=\{z \in \mathbb{C}:|z| \leq 1\}
$$

and

$$
C(\bar{D}):\{f: \bar{D} \rightarrow \mathbb{C}: f \text { is continuous on } D\} .
$$

Then, we propose the complex $q$-Post-Widder operators as follows:

$$
P_{n, q}^{*}(f ; z)=\frac{1}{[n]_{q} !} \int_{0}^{1 /(1-q)} f\left(\frac{z e^{i t}}{[n]_{q}}\right) t^{n} E_{q}(-q t) d_{q} t,
$$

where $z \in \bar{D}, n \in \mathbb{N}, f \in C(\bar{D})$ and $q \in(0,1)$.

It is not hard to see that if $f$ is a constant function on $\bar{D}$, say $f(z) \equiv c$, then we have, for every $n \in \mathbb{N}$ that $P_{n, q}^{*}(c ; z)=c$. Hence, the operators $P_{n, q}^{*}$ preserve the constant functions. In order to get some geometric properties of the operators $P_{n, q}^{*}$ in (6.1) we first need the following concepts.

Now define the subspace of $C(\bar{D})$ by

$$
A(\bar{D}):=\{f \in C(\bar{D}): f \text { is analytic on } D \text { with } f(0)=0\} .
$$

If $f \in C(\bar{D})$, then, as in Section 3, we consider the (first) modulus of continuity of $f$ on $\bar{D}$, denoted by $\omega(f, \delta)_{\bar{D}}, \delta>0$, as follows (see [11]):

$$
\omega(f ; \delta)_{\bar{D}}:=\sup \{|f(z)-f(w)|:|z-w| \leq \delta, z, w \in \bar{D}\} .
$$

It is easy to see that, for any $c, \delta>0$

$$
\omega(f ; c \delta)_{\bar{D}} \leq(1+c) \omega(f ; \delta)_{\bar{D}} .
$$

We obtain the following result.

Theorem 8. For each fixed $n \in \mathbb{N}$ and $q \in(0,1), P_{n, q}^{*}(A(\bar{D})) \subset A(\bar{D})$.

Proof. Let $n \in \mathbb{N}, f \in A(\bar{D})$ and $q \in(0,1)$ be fixed. Since $f(0)=0$, it follows from (6.1) that

$$
P_{n, q}^{*}(f ; 0)=\frac{1}{[n]_{q} !} \int_{0}^{1 /(1-q)} f(0) t^{n} E_{q}(-q t) d_{q} t=0 .
$$

Now we show that $P_{n, q}(f)$ is continuous on $\bar{D}$. To see this assume that $z,\left(z_{m}\right) \in$ $\bar{D}$ and that $\lim _{m} z_{m}=z$. Hence, we get from the definition of $\omega$ that

$$
\left|P_{n, q}^{*}\left(f ; z_{m}\right)-P_{n, q}^{*}(f ; z)\right|
$$




$$
\begin{aligned}
& \leq \frac{1}{[n]_{q} !} \int_{0}^{1 /(1-q)}\left|f\left(\frac{z_{m} e^{i t}}{[n]_{q}}\right)-f\left(\frac{z e^{i t}}{[n]_{q}}\right)\right| t^{n} E_{q}(-q t) d_{q} t \\
& \leq \frac{\omega\left(f, \frac{\left|z_{m}-z\right|}{[n]_{q}}\right)_{\bar{D}}}{[n]_{q} !} \int_{0}^{1 /(1-q)} t^{n} E_{q}(-q t) d_{q} t \\
& =\omega\left(f, \frac{\left|z_{m}-z\right|}{[n]_{q}}\right)_{\bar{D}} \\
& \leq \omega\left(f,\left|z_{m}-z\right|\right)_{\bar{D}}
\end{aligned}
$$

due to $[n]_{q} \geq 1$ for every $n \in \mathbb{N}$. Since $\lim _{m} z_{m}=z$, we may write that

$$
\lim _{m} \omega\left(f,\left|z_{m}-z\right|\right)_{\bar{D}}=0 .
$$

Due to the right continuity of $\omega(f, \cdot)$ at zero. Hence, we get

$$
\lim _{m} P_{n, q}^{*}\left(f ; z_{m}\right)=P_{n, q}(f ; z),
$$

which gives the continuity of $P_{n, q}^{*}(f)$ at the point $z \in \bar{D}$.

Finally, since $f \in A(\bar{D})$, we can write $f(z)=\sum_{k=1}^{\infty} a_{k} z^{k}$ for $z \in D$. Then, we get

$$
f\left(\frac{z e^{i t}}{[n]_{q}}\right)=\sum_{k=1}^{\infty} \frac{a_{k} z^{k} e^{i k t}}{[n]_{q}^{k}} .
$$

Since $\left|\frac{a_{k} e^{i k t}}{[n]_{q}^{k}}\right| \leq\left|a_{k}\right|$ for every $t \in \mathbb{R}$, the series in (6.3) is uniformly convergent with respect to $t \in \mathbb{R}$. Hence, we conclude that

$$
\begin{aligned}
P_{n, q}^{*}(f ; z) & =\frac{1}{[n]_{q} !} \int_{0}^{1 /(1-q)}\left(\sum_{k=1}^{\infty} \frac{a_{k} z^{k} e^{i k t}}{[n]_{q}^{k}}\right) t^{n} E_{q}(-q t) d_{q} t \\
& =\frac{1}{[n]_{q} !} \sum_{k=1}^{\infty} \frac{a_{k} z^{k}}{[n]_{q}^{k}}\left(\int_{0}^{1 /(1-q)} e^{i k t} t^{n} E_{q}(-q t) d_{q} t\right) \\
& =\sum_{k=1}^{\infty} \ell_{n, k} z^{k},
\end{aligned}
$$

where $\ell_{n, k}$ is given by

$$
\ell_{n, k}:=\frac{a_{k}}{[n]_{q} ![n]_{q}^{k}} \int_{0}^{1 /(1-q)} e^{i k t} t^{n} E_{q}(-q t) d_{q} t(k, n \in \mathbb{N}) .
$$


We should remark that

$$
\left|\ell_{n, k}\right| \leq\left|a_{k}\right| \text { for every } k, n \in \mathbb{N} .
$$

Therefore, for each $n \in \mathbb{N}$ and $f \in A(\bar{D})$, the function $P_{n, q}^{*}(f)$ has a Taylor series expansion whose Taylor coefficients are $\ell_{n, k}$ given by (6.4). Combining the above facts we obtain the desired result.

Now consider the following space:

$$
\begin{aligned}
B(\bar{D}):= & \{f \in C(\bar{D}): f \text { is analytic on } D \text { with } f(0)=1 \text { and } \\
& \operatorname{Re}[f(z)]>0 \text { for every } z \in D\} .
\end{aligned}
$$

Then we have the next result.

Theorem 9. For each fixed $n \in \mathbb{N}$ and $q \in(0,1), P_{n, q}^{*}(B(\bar{D})) \subset B(\bar{D})$.

Proof. Let $n \in \mathbb{N}, f \in B(\bar{D})$ and $q \in(0,1)$ be fixed. As in the proof of Theorem 8 , we see that $P_{n, q}^{*}(f)$ is analytic on $D$ and continuous on $\bar{D}$. Since $f(0)=1$, we easily get that

$$
P_{n, q}^{*}(f ; 0)=\frac{1}{[n]_{q} !} \int_{0}^{1 /(1-q)} f(0) t^{n} E_{q}(-q t) d_{q} t=1 .
$$

Finally, we may write that, for every $z \in D$,

$$
\operatorname{Re}\left[P_{n, q}^{*}(f ; z)\right]=\frac{1}{[n]_{q} !} \int_{0}^{1 /(1-q)} \operatorname{Re}\left[f\left(\frac{z e^{i t}}{[n]_{q}}\right)\right] t^{n} E_{q}(-q t) d_{q} t>0
$$

since $\operatorname{Re}\left[f\left(\frac{z e^{i t}}{[n]_{q}}\right)\right]>0$. Thus, the proof is completed.

If we consider the following space of Lipschitz class functions:

$$
\begin{gathered}
\operatorname{Lip}_{M}^{*}(\bar{D} ; \alpha):= \\
\left\{f: \bar{D} \rightarrow \mathbb{C} ;|f(z)-f(w)| \leq M|z-w|^{\alpha} \text { for every } z, w \in \bar{D}\right\}, \\
(\alpha \in(0,1] \text { and } M>0),
\end{gathered}
$$

we can get the next result.

Theorem 10. For each fixed $n \in \mathbb{N}$ and $q \in(0,1), P_{n, q}^{*}\left(\operatorname{Lip}_{M}^{*}(\bar{D} ; \alpha)\right) \subset \operatorname{Li} p_{M}^{*}(\bar{D} ; \alpha)$.

Proof. Let $f \in \operatorname{Lip}_{M}^{*}(\bar{D} ; \alpha)$. Then, for every $z, w \in \bar{D}$, we observe that

$$
\left|P_{n, q_{n}}^{*}(f ; z)-P_{n, q}^{*}(f ; w)\right| \leq \frac{1}{[n]_{q} !} \int_{0}^{1 /(1-q)}\left|f\left(\frac{z e^{i t}}{[n]_{q}}\right)-f\left(\frac{w e^{i t}}{[n]_{q}}\right)\right| t^{n} E_{q}(-q t) d_{q} t
$$




$$
\begin{aligned}
& \leq \frac{M}{[n]_{q} !} \int_{0}^{1 /(1-q)}\left|\frac{z e^{i t}}{[n]_{q}}-\frac{w e^{i t}}{[n]_{q}}\right|^{\alpha} t^{n} E_{q}(-q t) d_{q} t \\
& =\frac{M|z-w|^{\alpha}}{[n]_{q}^{\alpha}} \\
& \leq M|z-w|^{\alpha},
\end{aligned}
$$

which completes the proof.

Using the definition of $\omega(f ; \delta)_{\bar{D}}$ for $f \in C(\bar{D})$ and $\delta>0$, we obtain the following global smoothness preservation for the operators $P_{n, q}^{*}$ given by (6.1).

Theorem 11. For each fixed $n \in \mathbb{N}, q \in(0,1)$ and $f \in C(\bar{D})$, we have

$$
\omega\left(P_{n, q}^{*}(f) ; \delta\right)_{\bar{D}} \leq \omega(f ; \delta)_{\bar{D}}
$$

Proof. Let $\delta>0, n \in \mathbb{N}, q \in(0,1)$ and $f \in C(\bar{D})$ be given. Assume that $z, w \in \bar{D}$ and $|z-w| \leq \delta$. Then, we have

$$
\begin{gathered}
\left|P_{n, q}^{*}(f ; z)-P_{n, q}^{*}(f ; w)\right| \leq \frac{1}{[n]_{q} !} \int_{0}^{1 /(1-q)}\left|f\left(\frac{z e^{i t}}{[n]_{q}}\right)-f\left(\frac{w e^{i t}}{[n]_{q}}\right)\right| t^{n} E_{q}(-q t) d_{q} t \\
\leq \omega\left(f ; \frac{|z-w|}{[n]_{q}}\right)_{\bar{D}} \leq \omega\left(f ; \frac{\delta}{[n]_{q}}\right)_{\bar{D}} \leq \omega(f ; \delta)_{\bar{D}} .
\end{gathered}
$$

Then, taking supremum over $|z-w| \leq \delta$, we conclude that

$$
\omega\left(P_{n, q}^{*}(f) ; \delta\right)_{\bar{D}} \leq \omega(f ; \delta)_{\bar{D}}
$$

whence the result.

Now we obtain an estimation with the help of $\omega(f ; \delta)_{\bar{D}}$ for the operators $P_{n, q}^{*}$ defined by (6.1).

Theorem 12. For each fixed $n \in \mathbb{N}, q \in(0,1)$ and $f \in C(\bar{D})$, we have

$$
\left\|P_{n, q}^{*}(f)-f\right\|_{\bar{D}} \leq 3 \omega\left(f ; \frac{1}{\sqrt{[n]_{q}}}\right)_{\bar{D}}
$$

for some (finite) positive constant $M$, where $\|\cdot\|_{\bar{D}}$ denotes the usual sup-norm on $\bar{D}$ as stated before.

Proof. Let $z \in \bar{D}$ and $f \in C(\bar{D})$ be fixed. We first observe that

$$
\left|P_{n, q}^{*}(f ; z)-f(z)\right| \leq \frac{1}{[n]_{q} !} \int_{0}^{1 /(1-q)}\left|f\left(\frac{z e^{i t}}{[n]_{q}}\right)-f(z)\right| t^{n} E_{q}(-q t) d_{q} t
$$




$$
\leq \frac{1}{[n]_{q} !} \int_{0}^{1 /(1-q)} \omega\left(f ;|z|\left|\frac{e^{i t}}{[n]_{q}}-1\right|\right)_{\bar{D}} t^{n} E_{q}(-q t) d_{q} t .
$$

Since, for every $z \in D$ and $t \geq 0$,

$$
|z|\left|\frac{e^{i t}}{[n]_{q}}-1\right| \leq \sqrt{\frac{2}{[n]_{q}^{2}}-\frac{2 \cos t}{[n]_{q}}} \leq \frac{2|\sin (t / 2)|}{\sqrt{[n]_{q}}} \leq \frac{2}{\sqrt{[n]_{q}}}
$$

we may write that

$$
\begin{aligned}
\left|P_{n, q}^{*}(f ; z)-f(z)\right| & \leq \frac{1}{[n]_{q} !} \omega\left(f ; \frac{2}{\sqrt{[n]_{q}}}\right)_{\bar{D}} \int_{0}^{1 /(1-q)} t^{n} E_{q}(-q t) d_{q} t \\
& =\omega\left(f ; \frac{2}{\sqrt{[n]_{q}}}\right)_{\bar{D}}
\end{aligned}
$$

Then, by (6.2) we get

$$
\left|P_{n, q}^{*}(f ; z)-f(z)\right| \leq 3 \omega\left(f ; \frac{1}{\sqrt{[n]_{q}}}\right)_{\bar{D}}
$$

Taking supremum over $z \in \bar{D}$ on the last inequality, the proof is completed.

Using the Lipschitz class functions, we also obtain the next estimation result for the operators $P_{n, q}^{*}$.

Theorem 13. For each fixed $n \in \mathbb{N}, q \in(0,1)$ and $f \in \operatorname{Lip}_{M}^{*}(\bar{D} ; \alpha)$, we have

$$
\left\|P_{n, q}^{*}(f)-f\right\|_{\bar{D}} \leq \frac{2^{\alpha} M}{[n]_{q}^{\alpha / 2}} .
$$

Proof. Let $z \in \bar{D}$ and $f \in \operatorname{Lip} p_{M}^{*}(\bar{D} ; \alpha)$ be given. Then, one can write that

$$
\begin{aligned}
\left|P_{n, q}^{*}(f ; z)-f(z)\right| & \leq \frac{1}{[n]_{q} !} \int_{0}^{1 /(1-q)}\left|f\left(\frac{z e^{i t}}{[n]_{q}}\right)-f(z)\right| t^{n} E_{q}(-q t) d_{q} t \\
& \leq \frac{M}{[n]_{q} !} \int_{0}^{1 /(1-q)}|z|^{\alpha}\left|\frac{e^{i t}}{[n]_{q}}-1\right|^{\alpha} t^{n} E_{q}(-q t) d_{q} t .
\end{aligned}
$$

By (6.5), we get

$$
\left|P_{n, q}^{*}(f ; z)-f(z)\right| \leq \frac{2^{\alpha} M}{[n]_{q}^{\alpha / 2}} .
$$

Taking supremum over $z \in \bar{D}$ in the last inequality, the proof is completed. 
Now replacing the fixed number $q \in(0,1)$ with a sequence $\left(q_{n}\right)$ from the interval $(0,1)$ which satisfies the conditions in $(4.1)$, we can also obtain a statistical approximation result for our complex operators defined by (6.1)

Theorem 14. Let $A:=\left[a_{j n}\right]$ be a nonnegative regular summability matrix, and let $\left(q_{n}\right)$ be a sequence from $(0,1)$ satisfying (4.1). Then, for every $f \in C(\bar{D})$, we have

$$
s t_{A}-\lim _{n}\left\|P_{n, q_{n}}^{*}(f)-f\right\|_{\bar{D}}=0 .
$$

Proof. Let $f \in C(\bar{D})$. Then, for a given $\varepsilon>0$, we may write from Theorem 11 that

$$
\begin{array}{r}
U:=\left\{n \in \mathbb{N}:\left\|P_{n, q_{n}}^{*}(f)-f\right\|_{\bar{D}} \geq \varepsilon\right\} \subseteq\left\{n \in \mathbb{N}: \omega\left(f ; \frac{1}{\sqrt{[n]_{q_{n}}}}\right)_{\bar{D}} \geq \frac{\varepsilon}{3}\right\} \\
=: V .
\end{array}
$$

Thus, for every $j \in \mathbb{N}$, we get

$$
\sum_{n \in U} a_{j n} \leq \sum_{n \in V} a_{j n}
$$

On the other hand, by the right continuity of $\omega(f,)_{\bar{D}}$ at zero, one can get from (4.1) that

which gives

$$
s t_{A}-\lim _{n} \omega\left(f ; \frac{1}{\sqrt{[n]_{q_{n}}}}\right)=0,
$$

$$
\lim _{j} \sum_{n \in V} a_{j n}=0 .
$$

Now taking limit as $j \rightarrow \infty$ in the both sides of (6.6) and also considering (6.7) we obtain that

$$
\lim _{j} \sum_{n \in U} a_{j n}=0
$$

which means that

$$
s t_{A}-\lim _{n}\left\|P_{n, q_{n}}^{*}(f)-f\right\|=0 .
$$

The proof is completed.

If we replace $A$ by the identity matrix in Theorem 14, then we get the following uniform approximation result.

Corollary 6. Let $\left(q_{n}\right)$ be a sequence from $(0,1)$ satisfying (4.7). Then, for every $f \in A(\bar{D})$, the sequence $\left\{P_{n, q_{n}}^{*}(f)\right\}_{n \in \mathbb{N}}$ is uniformly convergent to $f$ on $\bar{D}$.

Finally, we give a statistical approximation result on the space $L i p_{M}^{*}(\bar{D} ; \alpha)$. 
Theorem 15. Let $A:=\left[a_{j n}\right]$ be a nonnegative regular summability matrix, and let $\left(q_{n}\right)$ be a sequence from $(0,1)$ satisfying (4.1). Then, for every $f \in \operatorname{Lip}_{M}^{*}(\bar{D} ; \alpha)$, we have

$$
s t_{A}-\lim _{n}\left\|P_{n, q_{n}}^{*}(f)-f\right\|_{\bar{D}}=0 .
$$

Proof. Let $z \in \bar{D}$ and $f \in \operatorname{Li} p_{M}^{*}(\bar{D} ; \alpha)$. By Theorem 13 , we get, for every $\varepsilon>0$, that

$$
\left\{n \in \mathbf{N}:\left\|P_{n, q_{n}}^{*}(f)-f\right\|_{\bar{D}} \geq \varepsilon\right\} \subseteq\left\{n \in \mathbf{N}: \frac{1}{[n]_{q_{n}}} \geq\left(\frac{\varepsilon}{2^{\alpha} M}\right)^{2 / \alpha}\right\} .
$$

Thus, we have, for every $j \in \mathbb{N}$,

$$
\sum_{n:\left\|P_{n, q_{n}}^{*}(f)-f\right\|_{\bar{D}} \geq \varepsilon} a_{j n} \leq \sum_{n: \frac{1}{[n] q_{n}} \geq\left(\frac{\varepsilon}{2^{\alpha} M}\right)^{2 / \alpha}} a_{j n} .
$$

Now taking limit as $j \rightarrow \infty$ in and also using (4.7), we immediately obtain that

$$
\lim _{j} \sum_{n \in Z_{1}} a_{j n}=0
$$

which gives

$$
s t_{A}-\lim _{n}\left\|P_{n, q_{n}}^{*}(f)-f\right\|_{\bar{D}}=0 .
$$

Thus, the proof is completed.

The next result is a natural consequence of Theorem 15.

Corollary 7. Let $\left(q_{n}\right)$ be a sequence from $(0,1)$ satisfying $(4.7)$. Then, for every $f \in \operatorname{Lip} p_{M}^{*}(\bar{D} ; \alpha)$, the sequence $\left\{P_{n, q_{n}}^{*}(f)\right\}_{n \in \mathbb{N}}$ is uniformly convergent to $f$ on $\bar{D}$.

\section{REFERENCES}

[1] O. Agratini, "On a $q$-analogue of stancu operators," Cent. Eur. J. Math., vol. 8, no. 1, pp. 191-198, 2010.

[2] A. Altı n, O. Doğru, and M. A. Özarslan, "Rates of convergence of Meyer-König and Zeller operators based on q-integers," WSEAS Trans. Math., vol. 4, pp. 313-318, 2005.

[3] A. De Sole and V. G. Kac, "On integral representations of $q$-gamma and $q$-beta functions," Atti Accad. Naz. Lincei, Cl. Sci. Fis. Mat. Nat., IX. Ser., Rend. Lincei, Mat. Appl., vol. 16, no. 1, pp. 11-29, 2005.

[4] R. A. DeVore and G. G. Lorentz, Constructive approximation, ser. Grundlehren der Mathematischen Wissenschaften. Berlin: Springer-Verlag, 1993, vol. 303.

[5] Z. Ditzian and V. Totik, Moduli of smoothness, ser. Springer Series in Computational Mathematics. New York: Springer- Verlag, 1987, vol. 9.

[6] O. Doğru and O. Duman, "Statistical approximation of Meyer-König and Zeller operators based on q-integers," Publ. Math., vol. 68, no. 1-2, pp. 199-214, 2006.

[7] O. Doğru and M. Örkcü, "Statistical approximation by a modification of $q$-Meyer-König and Zeller operators," Appl. Math. Lett., vol. 23, no. 3, pp. 261-266, 2010.

[8] H. Fast, "Sur la convergence statistique," Colloq. Math., vol. 2, pp. 241-244, 1951. 
[9] A. R. Freedman and J. J. Sember, "Densities and summability," Pac. J. Math., vol. 95, pp. 293305, 1981.

[10] A. D. Gadjiev and C. Orhan, "Some approximation theorems via statistical convergence," Rocky Mt. J. Math., vol. 32, no. 1, pp. 129-138, 2002.

[11] S. G. Gal, Shape-preserving approximation by real and complex polynomials. Basel: Birkhäuser, 2008.

[12] S. G. Gal and V. Gupta, "Approximation of vector-valued functions by $q$-Durrmeyer operators with applications to random and fuzzy approximation," An. Univ. Oradea, Fasc. Mat., vol. 16, pp. 233-240, 2009.

[13] V. Gupta and C. Radu, "Statistical approximation properties of $q$-Baskakov-Kantorovich operators," Cent. Eur. J. Math., vol. 7, no. 4, pp. 809-818, 2009.

[14] F. H. Jackson, "A generalisation of the functions $\Gamma(n)$ and $x^{n}$," Lond. R. S. Proc., vol. 74, pp. 64-72, 1904.

[15] F. H. Jackson, “On $q$-definite integrals," Quart. J., vol. 41, pp. 193-203, 1910.

[16] H. Karsli and V. Gupta, "Some approximation properties of $q$-Chlodowsky operators," Appl. Math. Comput., vol. 185, no. 1, pp. 220-229, 2008.

[17] H. Koelink and T. Koornwinder, " $q$-special functions, a tutorial," in Deformation theory and quantum groups with applications to mathematical physics, Proc. AMS-IMS-SIAM Jt. Summer Res. Conf., Amherst/MA (USA) 1990, ser. Contemp. Math. Providence, RI: American Mathematical Society, 1992, vol. 134, pp. 141-142.

[18] A. Lupas, "A q-analogue of the Bernstein operator," Prepr., "Babeş-Bolyai" Univ., Fac. Math., Res. Semin., vol. 9, pp. 85-92, 1987.

[19] N. I. Mahmudov and P. Sabancigil, " $q$-parametric Bleimann Butzer and Hahn operators," J. Inequal. Appl., vol. 2008, p. 15, 2008.

[20] N. I. Mahmudov, "Korovkin-type theorems and applications," Cent. Eur. J. Math., vol. 7, no. 2, pp. 348-356, 2009.

[21] S. Ostrovska, " $q$-Bernstein polynomials and their iterates," J. Approximation Theory, vol. 123, no. 2, pp. 232-255, 2003.

[22] S. Ostrovska, "On the Lupaş $q$-analogue of the Bernstein operator," Rocky Mt. J. Math., vol. 36, no. 5, pp. 1615-1629, 2006.

[23] S. Ostrovska, "Positive linear operators generated by analytic functions," Proc. Indian Acad. Sci., Math. Sci., vol. 117, no. 4, pp. 485-493, 2007.

[24] M. A. Özarslan, “ $q$-Laguerre type linear positive operators," Stud. Sci. Math. Hung., vol. 44, no. 1, pp. 65-80, 2007.

[25] G. M. Phillips, "On generalized Bernstein polynomials," in Approximation and optimization. Proceedings of ICAOR: international conference, Cluj-Napoca, Romania, July 29-August 1, 1996, D. D. Stancu, Ed. Cluj-Napoca: Transilvania Press, 1997, vol. 1, pp. 335-340.

[26] C. Radu, "On statistical approximation of a general class of positive linear operators extended in q-calculus," Appl. Math. Comput., vol. 215, no. 6, pp. 2317-2325, 2009.

[27] L. Rempulska and M. Skorupka, "The Voronovskaya-type theorems for Post-Widder and Stancu operators," Int. J. Pure Appl. Math., vol. 38, no. 4, pp. 467-480, 2007.

[28] T. Trif, "Meyer-König and Zeller operators based on the $q$-integers," Rev. Anal. Numér. Théor. Approx., vol. 29, no. 2, pp. 221-229, 2000. 
Authors' addresses

Zafer Ünal

Near East University, Faculty of Arts and Sciences, Department of Mathematics, Lefkoşa, Mersin 10, Turkey

E-mail address: zunaleneu.edu.tr

Mehmet Ali Özarslan

Eastern Mediterranean University, Faculty of Arts and Sciences, Department of Mathematics, Gazimagusa, Mersin 10, Turkey

E-mail address: mehmetali.ozarslan@emu.edu.tr

\section{Oktay Duman}

TOBB Economics and Technology University, Faculty of Arts and Sciences, Department of Mathematics, Söğütözü TR-06530, Ankara, Turkey

E-mail address: odumandetu.edu.tr 